\title{
The art and science of biobanking
}

\author{
Bartha Maria Knoppers - Thomas J. Hudson
}

Received: 5 July 2011/ Accepted: 5 July 2011/Published online: 21 July 2011

(C) Springer-Verlag 2011

The inspiration and impetus for this special issue on "Biobanking" came after over a decade of real world experience by the editors in the funding, building, and roll-out of biobanks around the world. Biobanks have been defined as "an organized collection of human biological material and associated information stored for one or more research purposes" (http://www.p3gobservatory.org/lexicon/list.htm). Our experience includes involvement in national projects such as the Canadian Partnership for Tomorrow Project (http://www.partnershipfortomorrow.ca). On an international level, we built both the Public Population Project in Genomics (http://www.p3g.org)—which aims to harmonize and enhance the interoperability of 43 large, longitudinal studies - and more recently, the International Cancer Genome Consortium (http://www.icgc.org). It is incumbent upon us to share the lessons we have learned and the multidisciplinary perspectives gained through the emergence of these resources. Indeed, we maintain that this decade is

\section{B. M. Knoppers}

Centre of Genomics and Policy, McGill University and Genome Quebec Innovation Centre, Montreal, QC, Canada

\section{B. M. Knoppers $(\square)$}

Centre of Genomics and Policy, Faculty of Medicine,

Department of Human Genetics, McGill University,

740 Dr. Penfield Avenue, Rm 5214, Montreal,

QC H3A 1A4, Canada

e-mail: bartha.knoppers@mcgill.ca

\section{T. J. Hudson}

Ontario Institute for Cancer Research,

101 College Street, Toronto, ON, Canada

\section{T. J. Hudson}

Departments of Molecular Genetics and Medical Biophysics, University of Toronto, Toronto, ON, Canada epitomized by the emergence of such infrastructure science alongside discovery science (Schofield et al. 2010).

To that end, this special issue on biobanking opens with an overview of the scientific logic underpinning the creation of biobanks and describes what translation might mean for population biobanking research (Murtagh et al. 2011). The authors argue that biobank resources need to be embedded within health systems and that both policy makers and health care providers need to be involved early on in the process. Moreover, they consider biobanking to be "not just a meeting but an intersection of disciplines." Accordingly, "within biobanking there is clear evidence not only of transdisciplinary structures, but of the transdisciplinary generation of knowledge." There is no doubt that today the preoccupation of biobanks is becoming the provision of liberal, but secure access to data and samples. Hence, there is a need for reflexive governance in biobanking, the subject of the second paper in this issue.

Reflexive governance is described by Graeme Laurie as "both about partnership in governance in the face of future uncertainty and the facilitation of mutual learning for experience over time" (Laurie 2011). It encourages "a culture of cooperation and compliance rather than monitoring non-compliance." Three principles support this approach: integrity of purpose, proportionality of action, and reflexivity of approach. Presenting the UK Biobank as an example, he suggests some potential limitations to reflexive governance: (1) can participants or other stakeholders be involved? (2) can it work in the absence of hard law? and (3) can it work for other biobanks? "A 'hard-law' regulatory approach may not achieve the uniform regulatory framework for biobanks that scientists and researchers seek." Moreover, "if at the heart of the biobanking enterprise is the need to promote issues such as trust and the public interest, it is not clear that laws alone can deliver on this task." 
Biobanking includes not just prospective longitudinal studies, but also the use of residual tissues leftover after medical care. According to Riegman and van Veen (2011), the preference for an individual, explicit and signed informed consent is overdone for the use of residual tissues in medical research and is not always the preferred choice of individual countries. For the use of residual tissues, they consider an opt-out system as a balanced choice between autonomy and the public good, provided certain conditions are met: (1) full transparency (patients are informed that their tissue could be used for research), (2) tissue is coded-anonymous, (3) research is geared toward important health questions, (4) tissue ownership rests with not-for-profit research groups, and (5) protocols are subject to REB approval.

The Kiehntopf and Krawczak (2011) paper addresses the uncertainty faced by sharing between biobanks due to a dearth of international standardization in biobanking. High variation in practices exists for the breadth of donor consent, methods of access, intellectual property rights, preanalytical handling and storage of biological samples, data management, and shipping. The requirement of an explicit consent usually implies that any sample transfer to third parties is subject to major constraints. This causes uncertainty over the right to transfer the material in the first place, the scope of research permitted by collaborators, and intellectual property rights. Collaborators are then advised to clarify any residual uncertainty by means of bilateral contracts. Biobank partners also face uncertainty over the quality of biomaterial they receive. The authors suggest that biobanks should implement stringent quality control measures and that biobank partners should claim contractual warranties for the type and quality of the biomaterial they wish to acquire. Similar roadblocks face the international transfer of data.

Rapid developments in information technologies are leading to an unanticipated growth in the ability to share data across projects and national boundaries. There are already increased exchanges of data through the internet using "research portals". Jane Kaye (2011) argues that existing governance frameworks were not designed to oversee this new way of doing research, given that most research ethics committees are usually local, address single projects, and expect that future research uses of samples and data are known in advance of obtaining consent and ethics approval. Kaye proposes a move toward "digital" governance which takes into account global research networks. Digital governance will require new IT interfaces, databases, algorithms, and universal digital identifiers for patients, researchers and biobanks in order to align oversight mechanisms with data systems and research projects that are appropriate for the twenty-first century.

The protection of privacy and confidentiality drives the debate on the possible re-identification of biobank participants; "defining the effectiveness" and "actual reidentification risk" of de-identification strategies is necessary to develop ethically sound policy (Malin et al. 2011). Malin et al. postulate that modeling "a potential attacker's resources and knowledge" and assessing the risk posed by such attack models can help policy makers to "tailor datasharing policies in light of real information." This paper goes on to demonstrate how decision makers can model and measure privacy risks in the context of known threats: "context impacts risk." A re-identification risk assessment can be carried out by considering "the replicability, availability, and distinguishability of the health information." The paper then moves on from the measurement of risk to a discussion of risk mitigation. "Risk mitigation strategies specialized to health and genomic data tend to focus on strategies that are able to preserve data truthfulness. Two popular methods that address this requirement are generalization, which replaces values with more general, but semantically consistent values, and suppression, which removes values from the released data."

According to Wallace and Kent (2011), several societal changes and technological developments such as whole genome sequencing have led to a reconsideration of the "no return" of research results policy in longitudinal population biobanks. Genomics knowledge is becoming part of everyday language and medical practice; people are taking greater individual control of their health; a "climate of expectation" is being generated by personal genomics companies about the power of DNA-based diagnostics; novel, cost-effective communication technologies make the return of results more feasible; and continuing advances in genetic science are increasing the probability of researchers uncovering incidental findings. Thus, in the near future biobanks will have to reconsider their "no return" policies and choose between notifying the participants' physician, asking an oversight committee to decide, returning all information, or maintaining a "no return" policy. If the policy were to be changed, would all participants have to be reconsented? Most researchers accessing biobanks in the future may be using whole genome sequencing technologies even though most information gathered is still indecipherable (Hayeems et al. 2011). Irrespective, the possibility of incidental findings of clinical significance remains (Bredenoord et al. 2011). Perhaps for now, the focus should be on more creative ways to return general results as they emerge so as to have adequate time for a proper public discussion on the no longer so "incidental" nature of such results.

A subject not often discussed in the social, ethical and legal literature is that of children and biobanks. According to Hens et al. (2011), three core requirements inform international ethical guidelines on children and biobanking: permission from a legal representative; assent and dissent; 
and an evaluation of its risks, benefits and necessity. Two novel observations of the authors bear mentioning here. The first is that they see the principle of solidarity as an alternative to autonomy centered bioethics. Some fear that applying the principle to children (who cannot consent) would be equivalent to forcing them to participate. But the authors consider that under this approach, participation is a means for children to "learn how to cooperate very early in life." They concede, however, that the principle requires further development: "[i]t is difficult to lay down exactly what is meant by a duty to participate in medical research," especially in cases with minors as participants. Another observation addresses the return of results. Should parents be able to decide not to receive vital information on their children? The right not to know should not apply to parents for early-onset treatable or preventable diseases, but at the same time, they should not have access to each and every piece of information on their children. Researchers have a more stringent duty to return results for children.

Research is increasingly long term so the impact of the death of a participant has important legal and ethical ramifications. Tassé's (2011) paper discusses the legal and ethical frameworks that govern the secondary use of deceased participants' data and samples as well as the issue of the return of results to biological family members. Few international guidelines address the effects of death and even if they do, it is mainly in terms of substituted consent to research itself for a deceased person, not the rights of persons already enrolled in research who have died. Most international guidelines do not foresee the impact of death. Interestingly, American law considers deceased persons as "non humans," while European and Canadian guidelines do not. All, however, fail to distinguish between the rights of the living and deceased participants in the research milieu. There is also little guidance for secondary use of samples and return of research results to biological family members. Indeed, uncertainty reigns as concerns the "rights of family members or the estate over samples, data and research results of the deceased." The author filters her discussion through traditional ethical principles and concludes that the autonomy principle leads to no obvious trend in favor or against secondary use or the return of results, but the principles of beneficence, nonmaleficence and justice seem to weigh in favor of the living family authorizing such research or receiving results in the absence of any known wishes of the deceased.

Like the inclusion of children in biobanking, the possibility of biobank closure is an under-explored issue. Zawati et al. (2011) consider the ethical issues associated with biobank sale, insolvency, end of funding, or transfer of materials to other entities. Population and DTC testing companies are used as case studies in the authors' discussion of international and national normative documents addressing closure, internal policies of population biobanks, and DTC consumer genetic testing companies. They underscore the fact that the OECD's Guidelines on Human Biobanks and Genetic Research Databases (2009) state that biobanks should have a plan for unexpected discontinuations, transfer, and destruction of human biological materials and data but few countries do so. According to the authors, closure plans should be emphasized in the initial consent process and address the issues of storage of data and samples, privacy protections during transfer, maximizing the use of donated samples and data, and maintaining the trust of participants.

The Gottweis et al. (2011) paper on public perceptions of biobanks is framed in light of Lippman's "phantom public": the idea that public only comes into existence when people become interested in something. It surveys the current state of knowledge on public-biobank relations and concludes that within Europe there appears to be large variation in preferences. Indeed, "[p]eople clearly differentiate between biobanks depending on the type and purpose of the biobank." Institutional set-up is important, as most people expect that biobanks are run publicly. Moreover, commercialization and private interests affect perception. There are also national differences in the way the "public" takes into account the common good and individual interests (e.g., higher demand for feedback in countries without routine health checks) and in concerns about exploitation through international collaboration. They conclude that more research is still needed on the links between biobanks and their publics. Biobanks should also gather precise and solid knowledge on the distribution of preferences.

Data access agreements complement legal and ethical norms to ensure that biobank resources are used properly. The Joly et al. (2011) paper reviews different sanctions that can be used in the case of non-compliance with these agreements. While only a few breaches have been experienced to date, "sanctioning is not a trivial issue." Nevertheless, a review of literature, policies and agreements reveals a lack of information on possible sanctions. To date, however, there is a "vast preference for the application of community based sanctions as a means to resolve cases of non-compliance." This is "because of the potentially strong negative impact of legal recourses on users as well as the difficulties of obtaining such recourses in an international forum."

Finally, "[e]thical challenges raised by genomic medicine reflect an enduring problem in public health: the appropriate balancing of individual and collective values, rights and interests. Biobanking in the context of health genomics reflects a unique case study in this classical problem" (Meslin and Garba 2011). Meslin et al. suggest a human-rights perspective for the biobanking ethical-legal 
framework, which integrates the concept of collective rights with notions of the global public good and the common heritage of humanity. This framework "provides a useful tool for helping mitigate the regulatory hazards associated with privately-funding biobanking enterprises" in lower to middle income countries.

Advances in public health require deeper knowledge of genetic, environmental, psycho-social, and lifestyle factors that lead to the development and progression of chronic diseases. This requires access to very large and comprehensive biobanks and databases that serve as platforms for answering a myriad of research questions. The Human Genome Project has taught us that sharing resources, technologies, and information with a large community of scientists will accelerate discovery, often beyond the scope of what the primary data generators could do themselves. Already flagged by Murtagh et al. in the opening article of this issue, the challenge today is not "can we build it?" or "will citizens participate?" but rather "will these resources be adequately accessible by researchers?" The articles in this issue have covered a range of topics written by an international group of ethicists, scientists, sociologists, political scientists, epidemiologists, and lawyers. Hopefully, they will stimulate the need for continued development of guidelines, policies and frameworks. Not only will this ensure the privacy of human subjects providing samples and information to biobanks, but also increase the opportunities for new discoveries through the sharing of data with the scientific community in order to respect participants and serve society.

Acknowledgments The authors wish to thank Ma'n H. Zawati at the Centre of Genomics and Policy, McGill University for his invaluable support in the preparation of this Special Issue and Adrian Thorogood for his editorial assistance. B.M.K. is supported by a Canada Research Chair in Law and Medicine. T.J.H. is the recipient of a Senior Investigator Awards from the Ontario Institute for Cancer Research, through generous support from the Ontario Ministry of Research. B.M.K. and T.J.H. receive funding support for the Public Population Project in Genomics (P3G) from Genome Canada, Genome Quebec, and the European Commission.

\section{References}

Bredenoord AL, Onland-Moret NC, Van Delden JJM (2011) Feedback of individual genetic results to research participants: in favor of a qualified disclosure policy. Human Mutat. doi: 10.1002/humu.21518

Gottweis H, Chen H, Starkbaum J (2011) Biobanks and the phantom public. Hum Genet. doi:10.1007/s00439-011-1065-y

Hayeems RZ, Miller FA, Li L, Bytautas JP (2011) Not so simple: a quasi-experimental study of how researchers adjudicate genetic research results. Eur J Hum Genet 19:740-747

Hens K, Lévesque E, Dierickx K (2011) Children and biobanks: a review of the ethical and legal discussion. Hum Genet. doi: 10.1007/s00439-011-1031-8

Joly Y, Zeps N, Knoppers BM (2011) Genomic databases access agreements: legal validity and possible sanctions. Hum Genet. doi:10.1007/s00439-011-1044-3

Kaye J (2011) From single biobanks to international networks: developing digital governance. Hum Genet. doi:10.1007/s00439011-1063-0

Kiehntopf M, Krawczak M (2011) Biobanking and international interoperability: samples. Hum Genet. doi:10.1007/s00439-0111068-8

Laurie G (2011) Reflexive governance in biobanking: on the value of policy led approaches and the need to recognise the limits of law. Hum Genet. doi:10.1007/s00439-011-1066-x

Malin B, Loukides G, Benitez K, Clayton E (2011) Identifiability in biobanks: models, measures, and mitigation strategies. Hum Genet. doi:10.1007/s00439-011-1042-5

Meslin EM, Garba I (2011) Biobanking and public health: is a human rights approach the tie that binds? Hum Genet. doi:10.1007/ s00439-011-1061-2

Murtagh MJ, Demir I, Harris JR, Burton PR (2011) Realizing the promise of population biobanks: a new model for translation. Hum Genet. doi:10.1007/s00439-011-1036-3

Riegman P, van Veen EB (2011) Biobanking residual tissues. Hum Genet. doi:10.1007/s00439-011-1074-x

Schofield PN et al (2010) Sustaining the data and bioresource commons. Science 330:592-593

Tassé AM (2011) Biobanking and deceased persons. Hum Genet. doi: 10.1007/s00439-011-1049-y

Wallace SE, Kent A (2011) Population biobanks and returning individual research results: mission impossible or new directions? Hum Genet. doi:10.1007/s00439-011-1021-x

Zawati MH, Borry P, Howard HC (2011) Closure of population biobanks and direct-to-consumer genetic testing companies. Hum Genet. doi:10.1007/s00439-011-1019-4 